\title{
Crystal phase effects in Si nanowire polytypes and
}

\section{their homojunctions}

\author{
Michele Amato, ${ }^{* \dagger}$ Thanayut Kaewmaraya ${ }^{\dagger}$ Alberto Zobelli, ${ }^{\ddagger}$ Maurizia Palummo, ${ }^{\text {II }}$ \\ and Riccardo Rurali*,
}

Centre de Nanosciences et de Nanotechnologies, CNRS, Univ. Paris-Sud, Université Paris-Saclay, 91405 Orsay, France, Laboratoire de Physique des Solides, Univ. Paris-Sud, Université Paris-Saclay 91405 France, Dipartimento di Fisica, Università di Roma Tor Vergata, Via della Ricerca Scientifica 1, 00133 Roma, Italy and INFN, Laboratori Nazionali di Frascati, Via E. Fermi 40, I-00044 Frascati, Italy, and Institut de Ciència de Materials de Barcelona (ICMAB-CSIC), Campus de Bellaterra, 08193 Bellaterra, Barcelona, Spain

E-mail: michele.amato@u-psud.fr; rrurali@icmab.es

\begin{abstract}
Recent experimental investigations have confirmed the possibility to synthesize and exploit polytypism in group IV nanowires. Driven by these promising evidences, we use firstprinciples methods based on density-functional theory and many-body perturbation theory to investigate the electronic and optical properties of hexagonal-diamond and cubic-diamond $\mathrm{Si}$ NWs as well as their homojunctions. We show that hexagonal-diamond NWs are characterized by a more pronounced quantum confinement effect than cubic-diamond NWs. Furthermore,
\end{abstract}

\footnotetext{
*To whom correspondence should be addressed

†Université Paris-Sud

†Université Paris-Sud

IIUniversità di Roma

§Institut de Ciència de Materials de Barcelona
} 
they absorb more light in the visible region with respect to cubic-diamond ones and, for most of the studied diameters, they are direct band-gap materials. The study of the homojunctions reveals that the diameter has a crucial effect on the band alignment at the interface. In particular, at small diameters the band-offset is type-I while at experimentally relevant sizes the offset turns up to be of type-II. These findings highlight intriguing possibilities to modulate electron and hole separations as well as electronic and optical properties by simply modifying the crystal phase and the size of the junction.

Introduction -Crystal structure and interface engineering are acquiring an increasing importance in nanoscience because of their enormous potential to conceive new properties and functionalities. ${ }^{1}$ In the case of nanowires (NWs), the emergence of new stable polytypes of common semiconductors promises to have an important impact in materials design. ${ }^{1-5}$ At typical device operation conditions, semiconductors present a well defined crystal phase: $\mathrm{Si}$, Ge and GaAs have a zinc-blende structure while, for instance, $\mathrm{GaN}, \mathrm{ZnO}$ and $\mathrm{CdSe}$ have a wurtzite one. Some of these systems can have other bulk phases which are stable only under extreme temperature and pressure conditions. This is particularly true for group IV based materials for which the existence of phases other than the cubic-diamond cannot be achieved in standard conditions. ${ }^{6-8}$

Nevertheless, in group IV nanowires, the stability of novel polytypes -previously theoretically predicted ${ }^{9}$ but experimentally observed only very locally in the form of crystal imperfections ${ }^{10-13}$ is now supported by clear experimental evidences. ${ }^{14-19}$ For instance, Vincent et al. ${ }^{14}$ reported the synthesis of quasi-periodic allotrope Ge heterostructures of hexagonal-diamond (wurtzite structure with single atom type) and cubic-diamond domains. These systems were obtained by a straininduced phase transformation in which size effects (in particular the preferential nucleation of dislocations on the wire surface and the activation of unusual slip planes) play a crucial role by lowering the value of the stress required by the cubic to hexagonal transition. A similar approach was adopted by Qiu et al. ${ }^{17}$ to produce hexagonal Si nanoribbon with a thickness of 5-8 nm. More recently, Hauge et al. ${ }^{16}$ have obtained pure and stable hexagonal silicon nanoshells with a thickness between 5-170 $\mathrm{nm}$ by growing them on the top of hexagonal GaP NWs used as a template. ${ }^{20,21}$ 
This experimental scenario suggests that exploiting polytypism in group IV NWs could be an efficient way to enhance NWs optoelectronic performances while retaining the compatibility with existent Si technology. As regards as Si NWs, recent experimental and theoretical investigations seem to confirm this prediction: cathodoluminescence measurements on hexagonal-diamond Si NWs ${ }^{22}$ show that these structures can emit visible light with a higher efficiency than cubicdiamond NWs; on the other hand, results of ab initio calculations ${ }^{23}$ have demonstrated that strained hexagonal-diamond bulk Si could be employed as an active layer in photovoltaic devices with absorption properties that are more favorable than those of cubic-diamond $\mathrm{Si}$.

Furthermore, important progresses in the synthesis of nanowires make possible to create homojunctions, where two different polytypes of the same material are grown on top of each other. When the growth is along specific crystallographic directions (e.g. the cubic $\langle 111\rangle$ parallel to the hexagonal [0001]), interfaces are in principle well defined and abrupt due to the minimal lattice mismatch between the two phases. While larger values have been reported for different phases of III-V semiconductors, the in-plane lattice mismatch between cubic and hexagonal Si is within $0.5 \%$. This leads to a minor relaxation of atomic bond lengths at the interface, while the stacking sequence in the growth direction changes, as illustrated in the sketch of Figure 5 for a homojunction between cubic-diamond and hexagonal-diamond $\mathrm{Si}$. The possibility of growing such kind of systems is extremely fascinating, as such Si-polytype junctions can be used to encode advanced functionalities in nanowires, going beyond the properties of their pristine, homogeneous counterparts.

Despite the great interest in Si NWs polytypism, its scientific potential remains largely unexplored and a unified description of the main properties of these polytypes and their homojunctions is still lacking. While the electronic and optical properties of Si bulk polytypes have been extensively studied in the past, ${ }^{24-27}$ very little is known on the influence of size reduction on the physical properties of such systems.

In this Letter we investigate, by means of state-of-the-art ab initio calculations, the electronic structure and optical response of hexagonal-diamond and cubic-diamond Si NWs as well as their 
homojunctions. In a first step we show that quantum confinement effects are more pronounced for hexagonal-diamond NWs than for cubic-diamond NWs. ${ }^{28,29}$ Furthermore, we demonstrate that hexagonal-diamond wires have a higher absorption in the visible spectral region with respect to cubic-diamond wires of similar diameter. This effect is associated, for most of the studied diameters, with a smaller direct band-gap. In a second step we analyze Si NWs homojunctions and we determine that the band alignment between the two phases is strongly diameter-dependent. In particular, for ultra-thin diameters, the cubic segment acts as a single quantum dot leading to a type-I offset while for larger diameters, the band offset can be tuned and turns to be of type-II. Altogether, the combination of direct and indirect band-gap domains at different sections of the wires with the presence of type-II junctions suggests promising applications in photovoltaics.

To this end, we perform density-functional theory (DFT) calculations with the SIESTA code ${ }^{30,31}$ of bulk $\mathrm{Si}$ and nanowires of different diameters in both the cubic and the hexagonal phase. For the largest NWs considered the structural relaxation has been obtained within the Density Functional Tight Binding (DFTB), as implemented in the the $\mathrm{dftb}+\mathrm{code},{ }^{32}$ and the electronic structure of these pre-relaxed structures with the same DFT computational setup of the bulk and thinner wires. A higher level description of the electronic structure of pure hexagonal and cubic Si bulk and the thinner NWs has been obtained following two approaches: using the HSE06 hybrid functional where a part of Hartree-Fock exchange is mixed into the Hamiltonian to partially correct the selfinteraction error (as implemented in the VASP code ${ }^{33,34}$ ); using the GW method (as implemented in the Yambo code ${ }^{35}$ ) on top of DFT self-consistent and non self-consistent calculation performed with the quantum-espresso package. ${ }^{36}$ Both the HSE06 and the GW approach have been used satisfactorily in the past and yield a cubic Si bulk band-gap of 1.14 and $1.08 \mathrm{eV}$, respectively. ${ }^{37-39}$ Optical properties have been calculated at the indipendent particle level for NWs and including also excitonic effects for bulks, through the solution of the Bethe-Salpeter Equation (BSE). Full details of the computational methods can be found in the Supporting Information.

Electronic and Optical Properties of Pure Cubic and Hexagonal NWs-As a first step, we have studied quantum confinement in pure cubic and hexagonal nanowires. Recently, pure hexago- 
nal Si NWs have been realized, ${ }^{16}$ thus these results are interesting per se, besides having important implications for the homojunctions discussed below. The diameter dependence of the electronic band-gap for a cubic and a hexagonal nanowire is displayed in Figure 1. As expected from previously published results (see for instance Ref. ${ }^{28,29,40,41}$ ), both these curves obey to the power law dependence

$$
E_{\text {gap }}^{N W}=E_{\text {gap }}^{\text {bulk }}+C d^{-\alpha}
$$

where $C$ and $\alpha$ are fitting parameters and $E_{\text {gap }}^{\text {bulk }}$ is the band-gap of the bulk of the corresponding polytype $\left(\alpha^{\text {hexagonal }}=1.16, \alpha^{\text {cubic }}=1.34\right)$. The difference in the $\alpha$ values can be ascribed to the difference in the effective mass among the two phases (see Figure 2). This leads to an inversion in the relative band-gap width for the two phases. For very small diameters, hexagonal NWs have a larger band-gap than cubic NWs but the difference vanishes for diameters $\sim 3.5-4 \mathrm{~nm}$ and then it changes sign for bigger diameters. For very large NWs, when quantum confinement effects become negligible, band-gaps converge to the bulk value, which is $\sim 0.1-0.3 \mathrm{eV}$ larger for cubic than for hexagonal Si (depending on the computational framework: LDA, HSE06 or GW), in agreement with other theoretical studies. ${ }^{23,42}$ Notice that in quantum confined NWs the bandgap is essentially determined by the growth orientation and the diameter, while the shape of the cross-section, thus the wire faceting, plays a negligible role. ${ }^{43,44}$

Noteworthy, as clearly shown in Figure 2, the band-gap of hexagonal NWs is indirect for the smallest diameter considered, but it becomes direct at the $\Gamma$ point for all the remaining diameters. On the other hand, the band-gap of cubic NWs shows an opposite behavior where only ultra-thin nanowires present a direct band-gap (see Figure 2), as previously reported. ${ }^{38,39,45}$

Local and semi-local approximation of the exchange-correlation energy yield to the wellknown underestimation of the band-gap that bedevils the most common implementation of densityfunctional theory. Indeed, we obtain $0.63 \mathrm{eV}$ for cubic bulk $\mathrm{Si}$, which should be compared with the experimental value of $1.1 \mathrm{eV}$. To provide an improved description of the electronic structure of bulk and pure hexagonal and cubic Si NWs, we employed the HSE06 hybrid functional and 
the GW approximation formalisms. Due to the high computational cost required by these methods, we tackled only the extreme cases of the bulk systems and the smaller diameter NWs, 1.0 to $1.75 \mathrm{~nm}$ for HSE06, 1.0 to $1.25 \mathrm{~nm}$ for GW. Results are plotted in the inset of Figure 1 . We obtain a qualitative agreement with the DFT-LDA trend, i.e. small diameter hexagonal NWs having larger band-gap than cubic NWs and cubic bulk Si having a larger band-gap than hexagonal bulk Si. These results vouch for the reliability of the DFT-LDA results obtained for thicker NWs, where using these more sophisticated methods would be unpractical. Notice that fitting the GW results to the power law of 1 predicts as well an inversion of the band-gap widths, in agreement with DFT-LDA, though it occurs at a slightly smaller diameter.

Next, we computed the optical response within an IP-RPA framework. Crystal local fields have been neglected since in nanowires they yield negligible depolarization effects when light is polarized along the wire's axis. ${ }^{46}$ In Figure 3 we report the calculated $\varepsilon_{2}(\omega)$ for hexagonal-diamond (right panel, bottom line) and cubic-diamond (right panel, top line) nanowires with diameter up to $1.8 \mathrm{~nm}$. The corresponding spectra of the two bulk phases are also shown (left panel of Figure 3) both at IP-RPA (orange curve) and BSE (blue curve) level of approximation to verify the consistency of our results with those published in Ref. ${ }^{23}$ As for the bulk, it is clear that also nanowires with the hexagonal-diamond phase present larger absorption in the visible region with respect to the cubic-diamond phase with a bigger overlap close to the maximum of the solar reference spectrum, possibly suggesting an improved power conversion efficiency. A graphical representation of this behavior is clearly shown in Figure 4, where a comparison between the absorption coefficients of bulk cubic and bulk hexagonal $\mathrm{Si}$ is presented.

Band-offset in cubic/hexagonal NW homojunction-We now move our attention to hexagonalcubic homojunctions in Si NWs of different diameter. This kind of structures can now be grown through advanced synthetic techniques and recent experimental results hint that a short hexagonal segment could behave as an optically active quantum dot. ${ }^{47,48}$ We computed cubic-hexagonal Si junctions with a $[111]_{\text {cubic }} /[0001]_{\text {hex }}$ interface in NWs of diameters 1, 1.8, and $3.2 \mathrm{~nm}$ (see Figure 5). For all NWs considered, cubic regions contain three unit cells (corresponding to a $2.82 \mathrm{~nm}$ 
section along the growth direction) while hexagonal ones have five unit cells replicas (that amounts to a length of $3.14 \mathrm{~nm}$ ).

To characterize the band-offset at the cubic-hexagonal interface, we have projected the total density of states (DOS) on two slices of material conveniently far from the interface: one in the cubic region and one in the hexagonal region. The results obtained are displayed in Figure 6. For sufficiently narrow NW diameter, the band-offset is type-I, with both the valence and the conduction band edge states localized on the cubic side of the junction (top panel of Figure 6). This is still the case for the $1.8 \mathrm{~nm} \mathrm{NW}$ (middle panel of Figure 6), but not for the $3.2 \mathrm{~nm}$, where the band-offset is now type-II (bottom panel of Figure 6): the conduction band edge states are still localized on the cubic side of the junction, but the valence band, at variance with the thinner NWs discussed above, is now on the hexagonal side. The observed change in the band-offset can be qualitatively related to the dependence of the band-gap of the pure phases as a function of the diameter (Figure 1). At very small diameters the band-gap of the cubic phase is considerably smaller than the band-gap of the hexagonal phase, a necessary condition for creating a sink for both electrons and holes and, hence, a type-I alignment. When the diameter increases, the difference between the cubic and the hexagonal band-gap vanishes and it is much easier that small differences in the electron affinity of the two phases result in a type-II band-offset. Notice that these are only qualitative considerations and that a self-consistent calculation of the electronic structure of the homojunction at a given diameter is always needed to assess the electrostatics at the interface and the band-offset analysis. For instance, as we discuss below, in the limit of very large diameter it is the band-gap of the hexagonal phase to become smaller than that of the cubic phase, but the band-offset remains type-II.

Although very thin diameters of the order of those studied above can nowadays exceptionally be achieved, ${ }^{29}$ routinely grown NWs have diameters in the 50-100 nm range. For this reason, we now study the band-offset in the limit case of large diameters where quantum and dielectric confinement effects are negligible. These systems can be effectively modeled by the corresponding bulk and hexagonal phases. We construct a bulk homojunction of cubic and hexagonal Si and study the 
position of the band edges as a function of the distance from the interface. To do that we proceed like for Figure 6, but project on several slices of material. As it is shown in Figure 7, holes are completely localized on the hexagonal region of the junction while the cubic part contains electrons thus demonstrating a type-II offset. This particular band alignment evokes the physics of single isolated hexagonal stacking faults in bulk cubic silicon. Indeed, as demonstrated in both experimental and theoretical works (see for instance Refs. ${ }^{49-51}$ ), the presence of hexagonal stacking faults in cubic $\mathrm{Si}$ is responsible for the creation of shallow states within the gap, usually 0.1-0.15 eV above the valence band maximum. This occurrence was also confirmed in the case of $[111]_{\text {cubic }} /[0001]_{\text {hex }}$ and $\{115\} /\{3 \overline{3} 02\}$ Si superlattices by Raffy and coworkers. ${ }^{52}$ This means that the valence states at the $\Gamma$ point are located on the hexagonal side, but, since the indirect bandgap of the cubic-diamond phase is preserved, the junction presents a type-II band alignment. This conclusion is further confirmed by GW calculations which give the same type of band alignment with the offset of valence and conduction bands amounting to 0.25 and $0.2 \mathrm{eV}$ (to be compared with 0.2 and 0.17 obtained with DFT-LDA).

However, a word of caution is of order here: as clearly explained by Raffy et al., ${ }^{52}$ the accurate evaluation of the conduction band-offset in such junctions requires a very precise determination of the position of the conduction band minimum in the $\mathbf{k}$ space (the accuracy should be lower than few ten meV). This difficulty has led in the past to contradictory results. ${ }^{26,52,53}$ Here, we confirm qualitatively the results of Ref. ${ }^{52}$ which also predicted a type-II band-offset for hexagonal-cubic Si superlattices, but with a smaller offset in the conduction band. To double-check the robustness of our results, we also carried out the calculation of the bulk band-offset with the same planewave computational framework ${ }^{54}$ used in Ref. ${ }^{52}$ and obtained an excellent quantitative agreement with their results. ${ }^{55}$ These results agree well also with the values obtained within our localized basis function setup used throughout the paper and that allowed tackling directly NW junctions that involve a few thousands of atoms. The valence band-offset is in excellent quantitative agreement with the plane-wave results, while the conduction band-offset is somewhat smaller and the agreement is only qualitative ( $0.1 \mathrm{eV}$ with plane-wave, $0.2 \mathrm{eV}$ with localized basis). 
Conclusions-In this work we shed light on the electronic and optical properties of Si NWs polytypes and their homojunctions. By applying several quantum-mechanical simulation methods with increasing levels of accuracy, we have carefully investigated the electronic band-gap scaling of hexagonal-diamond and cubic-diamond NWs confirming the validity of the power law dependence on the diameter. Interestingly, hexagonal-diamond NWs, unlike the corresponding bulk system, ${ }^{23}$ have a direct band-gap for most of the diameters considered. Furthermore, they absorb more light in the visible region with respect to cubic-diamond ones.

The study of the junctions has shown that the diameter has a crucial effect on the band alignment at the interface. In particular, at small diameters the band-offset is type-I while at experimentally relevant sizes the offset turns up to be of type-II. This nanoscale effect suggests intriguing possibilities to modulate electron and holes separations as well as absorption and emission properties by simply modifying the size of the junction.

At ultrathin diameters, cubic inclusions in an hexagonal wire act as a quantum dot that, together with the observed direct band-gap, could be exploited for quantum photonics and opto-electronics applications.

Yet, at larger diameters, the higher absorption of hexagonal NWs towards the visible region (that could even be improved by strain $^{23}$ ) together with the demonstrated type-II band-offset suggests novel concepts in Si NWs photovoltaics. We argue that creating NWs homojunctions of hexagonal and cubic-diamond could be a way to increase the absorbed portion of the solar spectrum as well as to enhance, thanks to the particular band alignment, the electron-hole separation.

\section{Acknowledgement}

We thank L. Vincent for fruitful discussions. We acknowledge financial support by the Ministerio de Economía y Competitividad (MINECO) under grant FEDER-MAT2013-40581-P and the Severo Ochoa Centres of Excellence Program under Grant SEV-2015-0496 and by the Generalitat de Catalunya under grants no. 2014 SGR 301. M.A. and T.K. acknowledge support from the Nanodesign project "Nanoharvesting" funded by the IDEX Paris-Saclay (ANR-11-IDEX-0003-02). 
Part of this work was made possible thanks to the HPC resources of IDRIS under the allocation i2015097422 and i2016097531 made available by GENCI (Grand Equipement National de Calcul Intensif) and the computer resources, technical expertise, and assistance provided by the Red Española de Supercomputación. M.P. acknowledges the EC for the RISE Project No. CoExAN GA644076 and CINECA for ISCRA-B Project No.HP10B0LUWN, for computational resources. 


\section{References}

(1) Jacobsson, D.; Panciera, F.; Tersoff, J.; Reuter, M. C.; Lehmann, S.; Hofmann, S.; Dick, K. A.; Ross, F. M. Nature 2016, 531, 317-322.

(2) Algra, R. E.; Verheijen, M. A.; Borgström, M. T.; Feiner, L.-F.; Immink, G.; van Enckevort, W. J. P.; Vlieg, E.; Bakkers, E. P. A. M. Nature 2008, 456, 369-372.

(3) Fontcuberta i Morral, A. Nature 2016, 531, 308-309.

(4) Assali, S.; Gagliano, L.; Oliveira, D. S.; Verheijen, M. A.; Plissard, S. R.; Feiner, L. F.; Bakkers, E. P. A. M. Nano Lett. 2015, 15, 8062-8069.

(5) Wang, F.; Dong, A.; Buhro, W. E. Chem. Rev. 2016, DOI:10.1021/acs.chemrev.5b00701.

(6) Jennings, H.; Richman, M. Science 1976, 193, 1242-1243.

(7) Pirouz, P.; Chaim, R.; Dahmen, U.; Westmacott, K. Acta Metallurgica et Materialia 1990, $38,313-322$.

(8) Ackland, G. Phys. Stat. Sol. (b) 2001, 223, 361-368.

(9) Amato, M.; Rurali, R. Prog. Surf. Sci. 2016, 91, 1-28.

(10) Jeon, N.; Dayeh, S. A.; Lauhon, L. J. Nano Lett. 2013, 13, 3947-3952.

(11) Shin, N.; Chi, M.; Howe, J. Y.; Filler, M. A. Nano Lett. 2013, 13, 1928-1933.

(12) Lopez, F. J.; Givan, U.; Connell, J. G.; Lauhon, L. J. ACS Nano 2011, 5, 8958-8966.

(13) Shin, N.; Chi, M.; Filler, M. A. ACS Nano 2013, 7, 8206-8213.

(14) Vincent, L.; Patriarche, G.; Hallais, G.; Renard, C.; Gardès, C.; Troadec, D.; Bouchier, D. Nano Lett. 2014, 14, 4828-4836.

(15) Biswas, S.; Doherty, J.; Majumdar, D.; Ghoshal, T.; Rahme, K.; Conroy, M.; Singha, A.; Morris, M. A.; Holmes, J. D. Chemistry of Materials 2015, 27, 3408-3416. 
(16) Hauge, H. I. T.; Verheijen, M. A.; Conesa-Boj, S.; Etzelstorfer, T.; Watzinger, M.; Kriegner, D.; Zardo, I.; Fasolato, C.; Capitani, F.; Postorino, P.; Kölling, S.; Li, A.; Assali, S.; Stangl, J.; Bakkers, E. P. A. M. Nano Lett. 2015, 15, 5855-5860.

(17) Qiu, Y.; Bender, H.; Richard, O.; Kim, M.-S.; Van Besien, E.; Vos, I.; de ten Broeck, M. d. P.; Mocuta, D.; Vandervorst, W. Sci. Rep. 2015, 5, 12692.

(18) Fabbri, F.; Rotunno, E.; Lazzarini, L.; Cavalcoli, D.; Castaldini, A.; Fukata, N.; Sato, K.; Salviati, G.; Cavallini, A. Nano Lett. 2013, 13, 5900-5906.

(19) Smith, B. E.; Zhou, X.; Roder, P. B.; Abramson, E. H.; Pauzauskie, P. J. J. Appl. Phys. 2016, $119,185902$.

(20) Algra, R. E.; Hocevar, M.; Verheijen, M. A.; Zardo, I.; Immink, G. G. W.; van Enckevort, W. J. P.; Abstreiter, G.; Kouwenhoven, L. P.; Vlieg, E.; Bakkers, E. P. A. M. Nano Lett. 2011, $11,1690-1694$.

(21) Conesa-Boj, S.; Hauge, H. I. T.; Verheijen, M. A.; Assali, S.; Li, A.; Bakkers, E. P. A. M.; i Morral, A. F. Nano Lett. 2015, 15, 2974-2979.

(22) Fabbri, F.; Rotunno, E.; Lazzarini, L.; Fukata, N.; Salviati, G. Sci. Rep. 2014, 4, 3603.

(23) Rödl, C.; Sander, T.; Bechstedt, F.; Vidal, J.; Olsson, P.; Laribi, S.; Guillemoles, J.-F. Phys. Rev. B 2015, 92, 045207.

(24) Joannopoulos, J.; Cohen, M. L. Phys. Rev. B 1973, 7, 2644.

(25) Joannopoulos, J.; Cohen, M. L. Phys. Rev. B 1973, 8, 2733.

(26) Raffy, C.; Furthmüller, J.; Bechstedt, F. Phys. Rev. B 2002, 66, 075201.

(27) De, A.; Pryor, C. E. J. Phys.: Condens. Matter 2014, 26, 045801.

(28) Rurali, R. Rev. Mod. Phys. 2010, 82, 427-449. 
(29) Ma, D. D. D.; Lee, C. S.; Au, F. C. K.; Tong, S. Y.; Lee, S. T. Science 2003, 299, 1874-1877.

(30) Soler, J. M.; Artacho, E.; Gale, J. D.; García, A.; Junquera, J.; Ordejón, P.; Sánchez-Portal, D. J. Phys.: Condens. Matter 2002, 14, 2745-2779.

(31) Artacho, E.; Anglada, E.; Diéguez, O.; Gale, J. D.; García, A.; Junquera, J.; Martin, R. M.; Ordejón, P.; Pruneda, J. M.; Sánchez-Portal, D.; Soler, J. M. Journal of Physics: Condensed Matter 2008, 20, 064208.

(32) Aradi, B.; Hourahine, B.; Frauenheim, T. J. Phys. Chem. A 2007, 111, 5678-5684.

(33) Kresse, G.; Furthmüller, J. Phys. Rev. B 1996, 54, 11169.

(34) Kresse, G.; Furthmüller, J. Comp. Mater. Sci. 1996, 6, 15-50.

(35) Marini, A.; Hogan, C.; Grüning, M.; Varsano, D. Comp. Phys. Comm. 2009, 180, 1392-1403.

(36) Giannozzi, P. et al. J. Phys.: Cond. Matter 2009, 21, 395502.

(37) Bruno, M.; Palummo, M.; Marini, A.; Del Sole, R.; Ossicini, S. Phys. Rev. Lett. 2007, 98, 036807.

(38) Zhao, X.; Wei, C. M.; Yang, L.; Chou, M. Y. Phys. Rev. Lett. 2004, 92, 236805.

(39) Rurali, R.; Aradi, B.; Frauenheim, T.; Gali, A. Phys. Rev. B 2007, 76, 113303.

(40) Palummo, M.; Hogan, C.; Ossicini, S. Phys. Chem. Chem. Phys. 2015, 17, 29085-29089.

(41) Amato, M.; Palummo, M.; Rurali, R.; Ossicini, S. Chem. Rev. 2014, 114, 1371-1412.

(42) De, A.; Pryor, C. E. J. Phys. Cond. Mat. 2014, 26, 045801.

(43) Ng, M.-F.; Zhou, L.; Yang, S.-W.; Sim, L. Y.; Tan, V. B. C.; Wu, P. Phys. Rev. B 2007, 76, 155435.

(44) Yao, D.; Zhang, G.; Li, B. Nano Lett. 2008, 8, 4557-4561. 
(45) Vo, T.; Williamson, A. J.; Galli, G. Phys. Rev. B 2006, 74, 045116.

(46) Bruneval, F.; Botti, S.; Reining, L. Phys. Rev. Lett. 2005, 94, 219701.

(47) Li, Y.; Liu, Z.; Lu, X.; Su, Z.; Wang, Y.; Liu, R.; Wang, D.; Jian, J.; Lee, J. H.; Wang, H.; Yu, Q.; Bao, J. Nanoscale 2015, 7, 1601-1605.

(48) Spirkoska, D. et al. Phys. Rev. B 2009, 80, 245325.

(49) Chou, M.; Cohen, M. L.; Louie, S. G. Phys. Rev. B 1985, 32, 7979.

(50) Mattheiss, L.; Patel, J. Phys. Rev. B 1981, 23, 5384.

(51) Weber, E.; Alexander, H. J. Phys. Paris (Colloq.) 1983, 44, 319-328.

(52) Raffy, C.; Furthmüller, J.; Wagner, J.-M.; Bechstedt, F. Phys. Rev. B 2004, 70, 195344.

(53) Murayama, M.; Nakayama, T. Phys. Rev. B 1994, 49, 4710.

(54) Kresse, G.; Joubert, D. Phys. Rev. B 1999, 59, 1758.

(55) Kaewmaraya, T.; Amato, M. in preparation. 


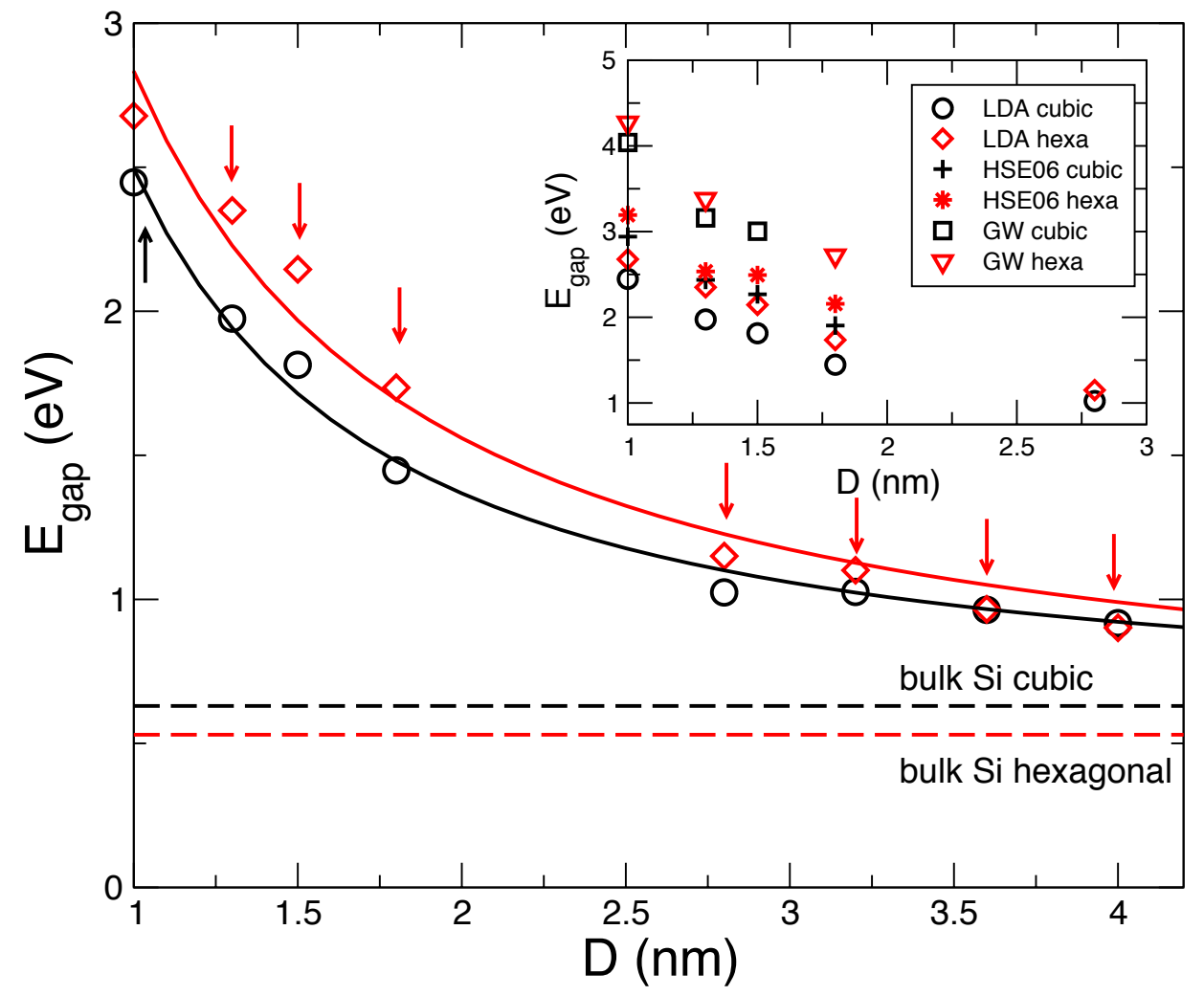

Figure 1: Bandgap of cubic (black dots) and hexagonal (red diamonds) Si NWs as a function of the diameter calculated with DFT-LDA; the arrows indicate the direct band-gaps. Results for the thinner wires calculated with HSE06 and GW are shown in the inset. Direct band-gaps have the maximum of the valence band and the minimum of the conduction band at $\Gamma$. In indirect band-gaps the minimum of the conduction band moves somewhere between $\Gamma$ and the zone boundary, while the maximum of the valence band remains at $\Gamma$. 

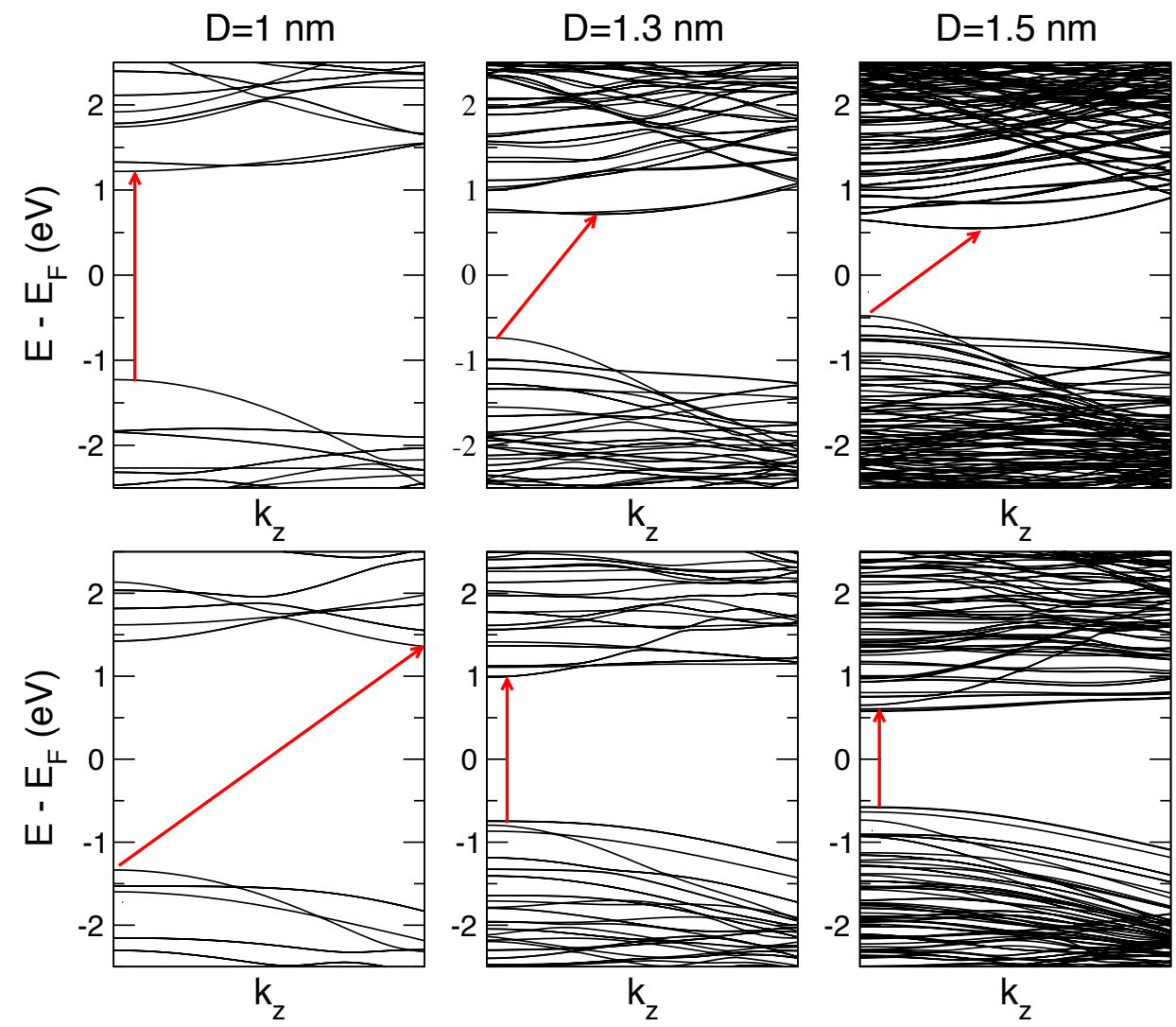

Figure 2: Band-structure diagram for (top row) cubic Si NWs and (bottom row) hexagonal Si NWs with a diameter of $1,1.3$, and $1.5 \mathrm{~nm}$. Red arrows indicate the fundamental band-gap, highlighting the indirect-direct and direct-indirect transitions as a function of the diameter. 
bulk Si

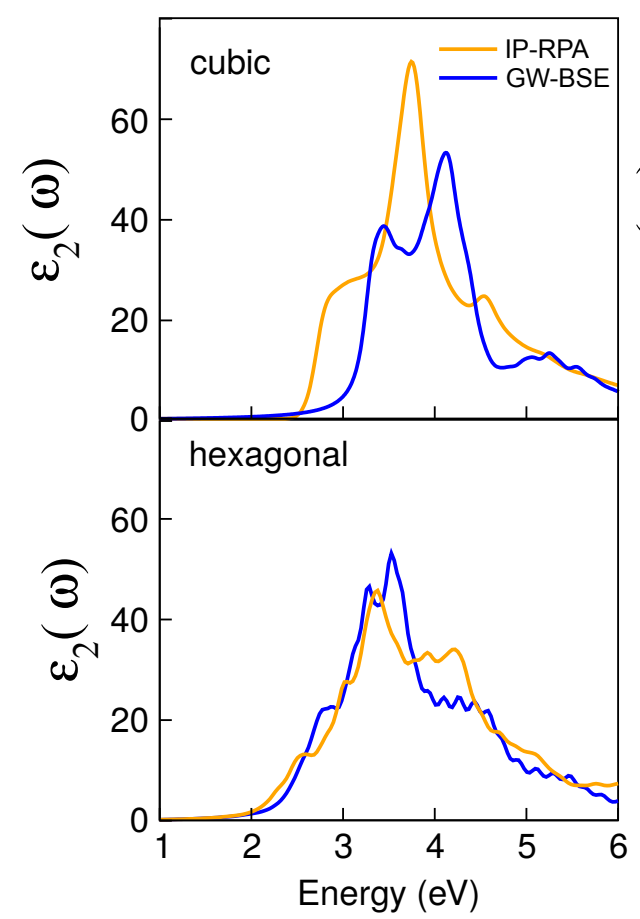

Si NWs

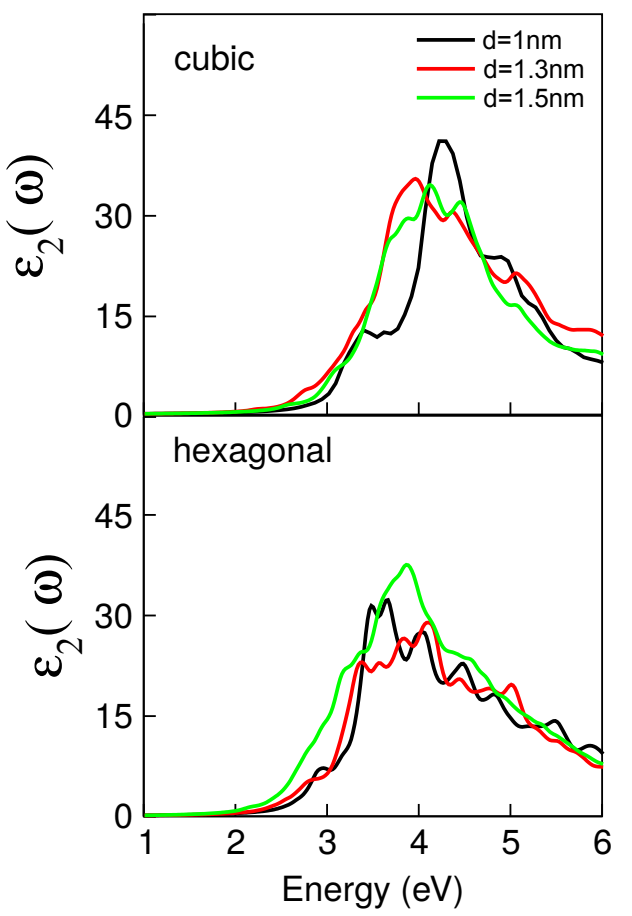

Figure 3: Left panel: absorption spectra of (top) cubic and (bottom) hexagonal bulk Si within an IP-RPA (orange line) and GW-BSE (blue line) framework. Right panel: absorption spectra of (top) cubic and (bottom) hexagonal Si NWs as a function of the diameter within an IP-RPA framework. The values have been properly normalized. However, due to the arbitrariness when it comes to estimate the diameter of the nanowires, comparisons between nanowires of different diameter or between nanowires and bulk are intended to be qualitative. 


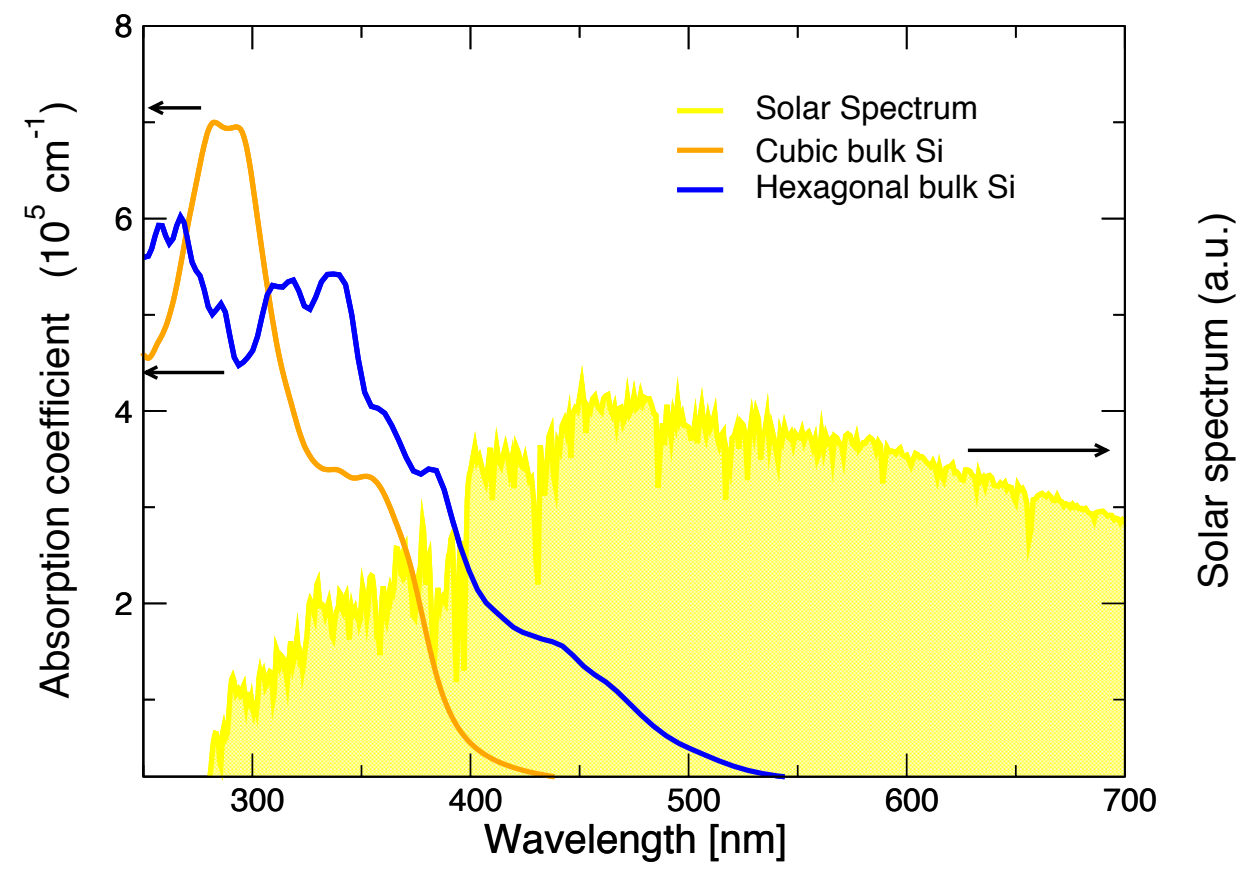

Figure 4: Absorption coefficient of bulk (orange line) cubic and (blue line) hexagonal Si within the GW-BSE framework. The AM 1.5 solar spectrum is shown for comparison. 

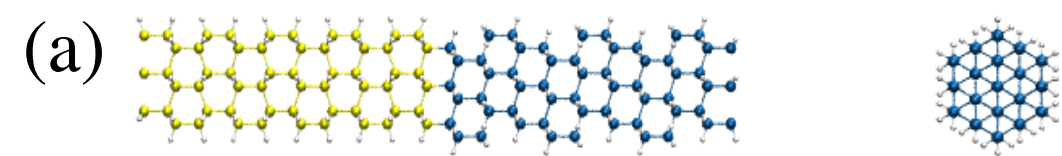

(b)
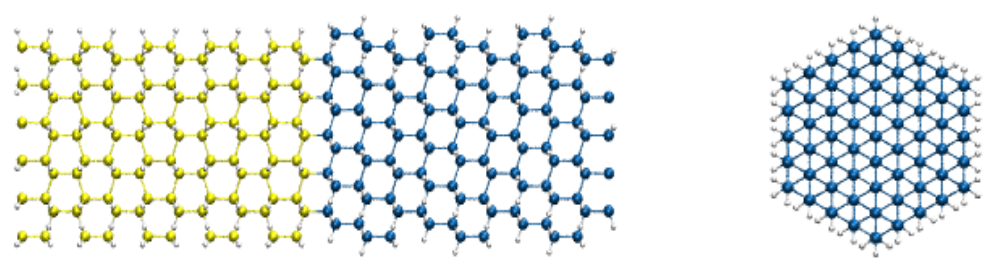

(c)
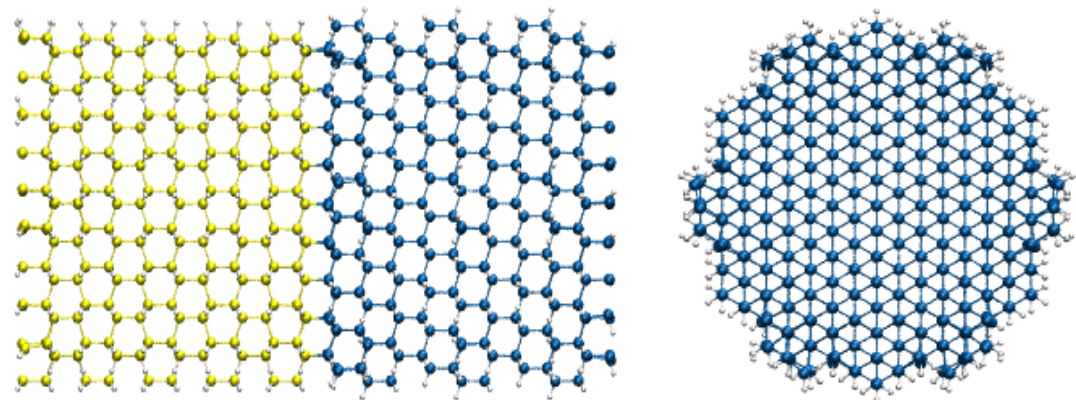

A B A B A B A B A B C A B C A B C A B

Figure 5: Cubic Si-hexagonal Si homojunctions in NWs of (a) 1, (b) 1.8, and (c) $3.2 \mathrm{~nm}$ diameter. $\mathrm{Si}$ atoms in the cubic (hexagonal) phase and hydrogen atoms are represented with blue (yellow) and white spheres, respectively. The stacking sequence along the wire axis is also indicated. Crosssection views of the NWs are displayed on the right-hand side of the figure. 


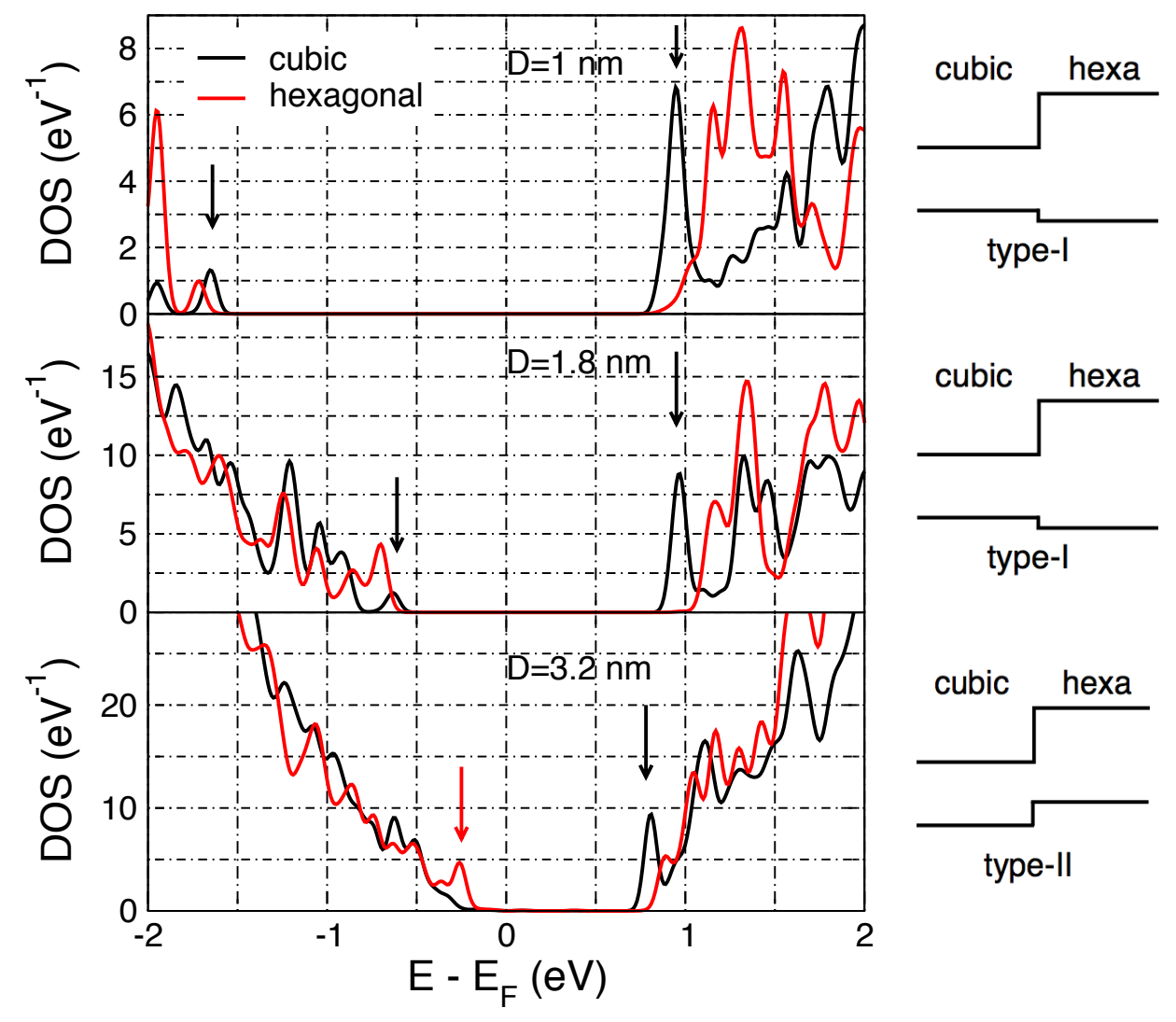

Figure 6: Projection of the density of states on atoms in the cubic and hexagonal phase for the homojunctions of Figure 5. Sketches on the band alignment type for each diameter are shown on the right-hand side. 


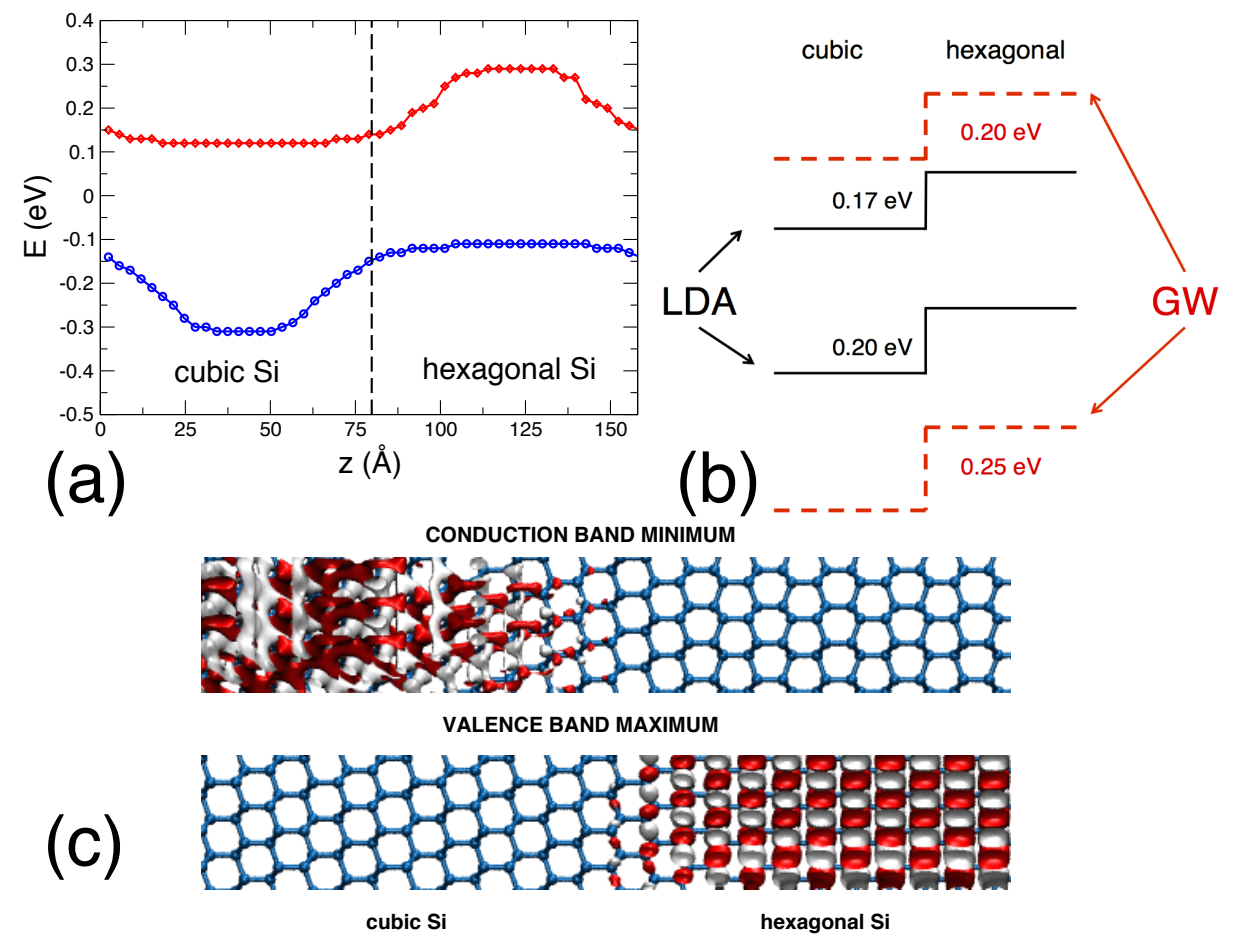

Figure 7: (a) Band-edges DFT-LDA eigenvalues as a function of the position in a bulk cubichexagonal homojunction. (b) GW correction to the band-offset scheme of panel (a). (c) Wavefunctions of the band-edge states of the bulk homojunction: the valence band maximum is localized on hexagonal $\mathrm{Si}$, while the conduction band minimum is localized on cubic $\mathrm{Si}$, thus the band alignment is type-II. 